Pacific Journal of Mathematic 


\title{
TOPOLOGIES FOR PROBABILISTIC METRIC SPACES
}

\author{
ROBERT M. TARDIFF
}

\begin{abstract}
Profile functions are used to construct a family of closure operators (in the sense of Čech) on a probabilistic metric space. Relationships among the various closure operators are considered, and products and quotients of probabilistic metric spaces are reexamined in light of this new topological structure.
\end{abstract}

O. Introduction. In their original paper [9], B. Schweizer and A. Sklar introduced a neighborhood structure for a probabilistic metric (PM) space, which, under suitable conditions, is metrizable [11]. However, the usefulness of this neighborhood structure is limited to those spaces in which, for every $\varepsilon>0$, there exist pairs of distinct points which have probabilities greater than $1-\varepsilon$ assigned to the event that the distance between them is less than $\varepsilon$. For example, $C$-spaces [10] do not have this property, with the result that the neighborhood structure of Schweizer and Sklar is discrete.

In [13] E. Thorp and in [6] R. Fritsche tried to overcome this difficulty, but, in so doing, each imposed a neighborhood structure on the PM space which, in general, failed to satisfy the following fundamental neighborhood axiom: If $N_{1}$ and $N_{2}$ are neighborhoods of a point $p$, then there is a neighborhood $N_{3}$ of $p$ such that $N_{3}$ is contained in the intersection of $N_{1}$ and $N_{2}$. Thus, each of their neighborhood structures did not yield a topology on the PM space, nor even a closure operator in the sense of Čech [2].

In this paper we use the profile functions introduced by Fritsche in [6] to construct a family of neighborhood structures for a PM space. With these neighborhood structures the difficulties incurred by Schweizer and Sklar are easily overcome. Furthermore, we show that for each profile function, the associated neighborhood structure satisfies the aforementioned neighborhood axiom, and hence, yields a closure operator on the PM space in the sense of Čech, and we determine sufficient conditions for this closure operator to be a closure operator in the sense of Kuratowski. We also study the relationships among the neighborhood structures determined by different profile functions and discuss the separation axioms in this context.

Next, we extend the work of R.J. Egbert [3] on products of PM spaces, the probabilistic diameter, and the probabilistic Hausdorff metric in two directions: First, we redefine these concepts in terms 
of triangle functions rather than $t$-norms [7]; second, we study their properties in terms of our new family of neighborhood structures rather than the neighborhood structure introduced by Schweizer and Sklar. Finally, we show that under suitable conditions, a profile function and the probabilistic Hausdorff metric can be used to define an equivalence relation on the points of a PM space which is related to the tolerance relation recently studied by B. Schweizer [8].

1. Preliminaries. The axiomatic characterization of a PM space is quite similar to that of a metric space. In such a space the range of the distance function is the set $\Delta^{+}$of one dimensional cumulative distribution functions on $[0, \infty)$, rather than the set of nonnegative real numbers; and a suitable semigroup operation defined on $\Delta^{+}$replaces the operation of addition in the triangle inequality.

More precisely, let

$$
\begin{gathered}
\Delta^{+}=\{F: R \rightarrow[0,1] \mid F \text { is nondecreasing, left-continuous, } \\
\text { and } F(0)=0\} .
\end{gathered}
$$

The set $\Delta^{+}$has a natural partial order; namely, $F \geqq G$ if and only if $F(x) \geqq G(x)$, for every $x$. The maximal element in $\Delta^{+}$with respect to this order is the distribution function

$$
\varepsilon_{0}(x)=\left\{\begin{array}{l}
0, \text { for } x \leqq 0 \\
1, \text { for } x>0
\end{array}\right.
$$

In [12] D.A. Sibley exhibited a natural metric for $\Delta^{+}$, called the modified Lévy metric $\mathscr{L}$ and showed that the metric space $\left(\Delta^{+}, \mathscr{L}\right)$ is compact and arc-wise connected. This metric can be defined as follows: for any $F$ and $G$ in $\Delta^{+}$and any $h>0$, let $A$ and $B$ denote the following properties:

$$
\begin{aligned}
& \text { (1.1) } A(F, G ; h) \Longleftrightarrow F(x-h)-h \leqq G(x), \text { for } x \in\left[0, \frac{1}{h}+h\right), \\
& \text { (1.2) } B(F, G ; h) \Longleftrightarrow F(x+h)+h \geqq G(x), \text { for } x \in\left[0, \frac{1}{h}\right) .
\end{aligned}
$$

Then,

$$
\mathscr{L}(F, G)=\inf \{h \mid A(F, G ; h) \text { and } B(F, G ; h)\} .
$$

DEFINITION 1.1. A probabilistic semi-metric space (briefly, a semi-PM space) is an ordered pair $(S, \mathscr{F})$, where $S$ is a set, and $\mathscr{F}$ is a mapping from $S \times S$ into $\Delta^{+}$such that for all pairs of points $p$ and $q$ in $S$ : 

(i) $\mathscr{F}(p, q)=\varepsilon_{0}$ if and only if $p=q$,
(ii) $\mathscr{F}(p, q)=\mathscr{F}(q, p)$.

The function $\mathscr{F}(p, q)$ is usually denoted by $F_{p q}$, and $F_{p q}(x)$, its value at $x$, is interpreted as the probability that the distance between $p$ and $q$ is less than $x$.

Definition 1.2. A two place function $\tau$ mapping $\Delta^{+} \times \Delta^{+}$into $\Delta^{+}$is called a triangle function if, for all $F, G$, and $H$ in $\Delta^{+}$

( $\alpha) \tau\left(F, \varepsilon_{0}\right)=F$,

( $\beta) \quad \tau(F, G) \geqq \tau(F, H)$ whenever $G \geqq H$,

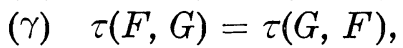

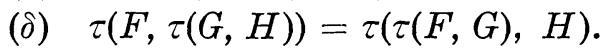

For any argument $x$, the value of the distribution function $\tau(F, G)$ at $x$ is denoted by $\tau(F, G ; x)$. A triangle function is continuous, if it is a continuous function from $\Delta^{+} \times \Delta^{+}$into $\Delta^{+}$, where $\Delta^{+}$is endowed with the topology induced by the modified Lévy metric, and $\Delta^{+} \times \Delta^{+}$, with the corresponding product topology.

There are many examples of triangle functions in the literature (see [7]). One is convolution. In addition, two families of triangle functions arise from $t$-norms [7]; i.e., suitable semigroups on the unit interval $[0,1]$, which satisfy conditions corresponding to $(\alpha)$ through $(\delta)$ of Definition 1.2. These are given by:

$$
\begin{gathered}
\tau_{T}(F, G ; x)=\sup _{u+v=x} T(F(u), G(v)) ; \\
\Pi_{T}(F, G ; x)=T(F(x), G(x)) ;
\end{gathered}
$$

where $T$ is a $t$-norm. For example, $\operatorname{Min}(a, b), \operatorname{Prod}(a, b)=a b$, and $T_{m}(a, b)=\operatorname{Max}(a+b-1,0)$ are all $t$-norms.

Definition 1.3. Let $(S, \mathscr{F})$ be a semi-PM space, and let $\tau$ be a triangle function. Then $(S, \mathscr{F})$ is a probabilistic metric space under $\tau$ (briefly, a PM space) if, for every triple of points $p, q$, and $r$ in $S$ :

$$
F_{p q} \geqq \tau\left(F_{p r}, F_{r q}\right) \quad \text { (triangle inequality). }
$$

If this is the case, then we say that $(S, \mathscr{F}, \tau)$ is a PM space.

Finally, we collect the results about closure spaces which will be needed in the sequel. The concept of a closure space is due to E. Cech, and the proofs of the various statements may be found in his book [2]. Many of the results may also be found in the work of M. Frechet [5] and A. Appert and Ky-Fan [1].

Definition 1.4. A closure space is a pair $(S, C)$, where $S$ is a set, and $C$ is a mapping from $\mathscr{P}(S)$, the power set of $S$, into itself 
such that

(1) $C(\square)=\square$,

(2) $A \subset C(A)$ for every $A \in \mathscr{P}(S)$,

(3) $C(A \cup B)=C(A) \cup C(B)$ for all $A, B \in \mathscr{P}(S)$.

Note that, in general, the mapping $C$, defined above, fails to be a closure operator in the sense of Kuratowski, since it is not required that $C(C(A))=C(A)$. A subset $A$ of $S$ is $C$-closed, if $C(A)=A$; and, $C$-open, if its complement $S \backslash A$ is $C$-closed. The interior of any subset $A$ of $S$, denoted by $\operatorname{Int}(A)$, is the set $S \backslash C(S \backslash A)$.

Theorem 1.5. Let $(S, C)$ be a closure space. Then the collection, $\mathscr{C}$, of all $C$-closed subsets of $S$ is closed under finite unions and arbitrary intersections.

Theorem 1.6. Let $(S, C)$ be a closure space. For each $A \in \mathscr{P}(S)$ let $\mathscr{B}_{A}=\{B \in \mathscr{C} \mid A \subset B\}$, and define $C^{*}(A)=\bigcap_{B \in \mathscr{B}_{A}} B$. Then $C^{*}$ is a Kuratowski closure operator; i.e., satisfies (1), (2) and (3) of Definition 1.4 and

$$
C^{*}\left(C^{*}(A)\right)=C^{*}(A) .
$$

Furthermore, if $A$ is C-closed, then $A$ is $C^{*}$-closed.

Thus, every closure space $(S, C)$ has a natural Kuratowski closure operator associated with it, and a topology in the usual sense. In this topology the closed sets are precisely those subsets of $S$ which are $C$-closed. It is easily shown that the inclusion $C(A) \varsubsetneqq C^{*}(A)$ is possible.

Closure spaces may also be characterized by their neighborhood structure:

Definition 1.7. Let $(S, C)$ be a closure space, and let $p \in S$ be given.

(1) A $C$-neighborhood of $p$ is any subset, $N$, of $S$ such that $p \in \operatorname{Int}(N)$.

(2) The C-neighborhood system at $p, \mathscr{N}_{c}(p)$, is the collection of all $C$-neighborhoods of $p$.

(3) A local base at $p$ for $\mathscr{N}_{c}(p)$ is any collection $\mathscr{U}$ of $C$-neighborhoods of $p$ which is equivalent to $\mathscr{N}_{c}(p)$.

(4) The C-neighborhood system, $\mathscr{N}_{C}$, is the collection $\mathrm{U}_{q \in S} \mathscr{N}_{C}(q)$.

(5) A base for $\mathscr{N}_{C}$ is any family, $\mathscr{U}$, of $C$-neighborhoods such that for each $p \in S$ there is a subfamily $\mathscr{C}(p)$ of $\mathscr{U}$ which is a local base at $p$. 
THEOREM 1.8. If $(S, C)$ is a closure space, then for each $p \in S$, $\mathscr{N}_{c}(p)$ satisfies the following three neighborhood axioms:

(nbd 1) The collection $\mathscr{N}_{c}(p)$ is not empty;

(nbd 2) For each $N \in \mathscr{N}_{c}(p), p \in N$;

(nbd 3) For every $N_{1}$ and $N_{2}$ in $\mathscr{N}_{0}(p)$, there is an $N_{3} \in \mathscr{N}_{c}(p)$ such that $N_{3} \subset N_{1} \cap N_{2}$.

To state a converse of Theorem 1.8 the following definition is needed:

DeFinition 1.9. Let $S$ be a nonempty set and suppose that for each $p \in S$ there is a collection $\mathscr{U}(p)$ of subsets of $S$. Then $C_{z}$ is the mapping from $\mathscr{P}(S)$ into $\mathscr{P}(S)$ given by:

$$
C_{z}(A)=\{q \in S \mid U \cap A \neq \square \text { for every } U \in \mathscr{U}(q)\} .
$$

THEOREM 1.10. Let $S$ be a nonempty set, and suppose that for each $p \in S$ there is a collection $\mathscr{\mathscr { C }}(p)$ of subsets of $S$ which satisfies (nbd 1), (nbd 2), and (nbd 3). Then $\left(S, C_{\varkappa}\right)$ is a closure space and, for each $p \in S, \mathscr{C}(p)$ is a local base for $N_{c_{2}}(p)$. In addition, if, for each $p \in S$, there is another collection $\mathscr{Y}(p)$ of subsets of $S$ which is equivalent to $\mathscr{\mathscr { C }}(p)$, then $C_{\mathfrak{L}}(A)=C_{\mathscr{r}}(A)$ for every $A \subset S$.

The importance of Definition 1.9 and Theorem 1.10 lies in the fact that together they yield a method for generating closure spaces. Indeed, this method will be used to induce a closure space structure on a semi-PM space. The next theorem yields necessary and sufficient conditions for a closure operator to be idempotent.

Theorem 1.11. Let $(S, C)$ be a closure space. The C-closure $C(A)$ of each $A \subset S$ is C-closed if and only if the following condition is satisfied:

(nbd 4) For each $p \in S$ and for each $N \in \mathscr{N}_{0}(p)$, there is a $V \in \mathscr{N}_{c}(p)$ such that $q \in V$ implies there is a $W \in \mathscr{N}_{c}(q)$ such that $W \subset N$.

2. A family of closure operators for semi-PM spaces. Let $(X, d)$ be a metric space. For each $h \geqq 0$ a closure operator $C_{h}$ for $X$ can be constructed in the following manner: For each $p \in X$ the $(h, \varepsilon)$-neighborhood of $p$ is the set $N_{p}(h, \varepsilon)=\{q \in S \mid d(p, q)<h+\varepsilon\}$. The h-neighborhood system at $p, \mathscr{N}_{h}(p)$, is the collection of all $(h, \varepsilon)$ neighborhoods at $p, \varepsilon>0$. For any $A \subset X$, let $C_{h}(A)=\{q \in$ $\left.X \mid N_{p}(h, \varepsilon) \cap A \neq \square, \in>0\right\}$. It is clear that for every $p$ in $X$, $\mathscr{N}_{h}(p)$ satisfies (nbd 1), (nbd 2), and (nbd 3); and hence, by Theorem 
1.10, $\left(S, C_{h}\right)$ is a closure space. Furthermore, in general, $\mathscr{N}_{h}(p)$ fails to satisfy (nbd 4).

In an analogous fashion, we shall show that if $(S, \mathscr{F})$ is a semi-PM space, then for each $\phi$ in $\Delta^{+}$, there is a closure operator $C_{\phi}$ for $S$. We begin with:

Definition 2.1. Let $(S, \mathscr{F})$ be a semi-PM space; let $\phi$ be in $\Delta^{+}$; and let $\varepsilon$ be a positive number. For each $p$ in $S$ :

(1) The $(\phi, \varepsilon)$-neighborhood of $p$ is the set

$$
\mathscr{N}_{p}^{\sigma}(\phi, \varepsilon)=\left\{q \in S \mid F_{p q}(x+\varepsilon)+\varepsilon \geqq \phi(x), \text { for } x \in[0,1 / \varepsilon)\right\} \text {. }
$$

(2) The $(\phi, \varepsilon)$-neighborhood system at $p$ is the collection

$$
\mathscr{N}_{\phi}^{\mathscr{T}}(p)=\left\{\mathscr{N}_{p}^{\mathscr{F}}(\phi, \varepsilon) \mid \varepsilon>0\right\} \text {. }
$$

(3) The $(\phi, \varepsilon)$-neighborhood system is the collection

$$
\left\{\mathscr{N}_{\phi}^{\pi}(p) \mid p \text { in } S\right\} \text {. }
$$

When there is no ambiguity, the " $\mathscr{F}$ " in the definitions of $\mathscr{N}_{p}^{\sigma}(\phi, \varepsilon), \mathscr{N}_{\phi}^{\mathscr{F}}(p)$, and $\mathscr{N}_{\phi}^{\mathscr{T}}$ will be suppressed.

Note that $q \in N_{p}(\phi, \varepsilon)$ if and only if $B\left(F_{p q}, \phi, \varepsilon\right)$ (viz. (1.2)). The function $\phi$ is called a profile function [6], and its value at $x, \phi(x)$, is interpreted as the maximum probability assignable to the event that the distance between $p$ and $q$ is less than $x$.

Theorem 2.2. Let $(S, \mathscr{F})$ be a semi-PM space; let $\phi$ and $\psi$ be profile functions; and, for any $A \subset S$, let

$$
C_{\phi}(A)=\left\{p \in S \mid N_{p}(\phi, \varepsilon) \cap A \neq \square \text {, for every } \varepsilon>0\right\} .
$$

Then $\left(S, C_{\phi}\right)$ is a closure space, having a countable local base at each $p \in S$. Moreover, if $\phi \geqq \psi$, then $C_{\phi}(A) \subset C_{\psi}(A)$ for every $A \subset S$.

Proof. Let $p \in S$. Since $F_{p p}=\varepsilon_{0}$, (nbd 1) and (nbd 2) follow immediately. Next, if $q \in N_{p}(\phi, \delta)$ and if $\eta>\delta$, then

$$
F_{p q}(x+\eta)+\eta \geqq F_{p q}(x+\delta)+\delta \geqq \phi(x), \quad \text { for } x \in\left[0, \frac{1}{\delta}\right) \supset\left[0, \frac{1}{\eta}\right) ;
$$

whence $q \in N_{p}(\phi, \eta)$ and $N_{p}(\phi, \delta) \subset N_{p}(\phi, \eta)$. It follows that for any $\varepsilon_{1}, \varepsilon_{2}>0$,

$$
N_{p}\left(\phi, \operatorname{Min}\left(\varepsilon_{1}, \varepsilon_{2}\right)\right) \subset N_{p}\left(\phi, \varepsilon_{1}\right) \cap N_{p}\left(\phi, \varepsilon_{2}\right),
$$

and (nbd 3) holds. Thus, by Theorem 1.10, $\left(S, C_{\phi}\right)$ is a closure space. Furthermore, the family $\mathscr{V}(p)=\left\{N_{p}(\phi, r) \mid r\right.$ is rational $\}$ is a 
countable local base for $\mathscr{N}_{\phi}(p)$. Lastly, if $\phi \geqq \psi$, then $N_{p}(\phi, \varepsilon) \subset$ $N_{p}(\psi, \varepsilon)$ for every $\varepsilon>0$, and thus, by (2.1), $C_{\phi}(A) \subset C_{\psi}(A)$ for every $A \subset S$.

Thus, if $(S, \mathscr{F})$ is a semi-PM space, then each profile function $\phi$ induces a closure operator $C_{\phi}$ on $S$; and hence, a natural topology on $S$ in the sense of Theorem 1.6. This is an improvement over Theorem 3.1 of [6], since the function $\phi$ is not restricted in any way.

In order to develop some of the deeper properties of the closure spaces $\left(S, C_{\phi}\right)$, we need several lemmas. We begin with:

Definition 2.3. Let $F \in \Delta^{+}$, and let $h \in[0,1]$ be given. Then $F^{h}$ is the function in $\Delta^{+}$defined by:

$$
\left\{\begin{array}{cl}
0, & \text { for } x \leqq 0 ; \\
F^{h}(x)=\operatorname{Min}[F(x+h)+h, 1], & \text { for } x \in\left(0, \frac{1}{h}\right] ; \\
1, & \text { for } x>\frac{1}{h} .
\end{array}\right.
$$

(Note that $F^{0}=F$ and $F^{1}=\varepsilon_{0}$.)

It is easily shown that $\mathscr{L}\left(F, F^{h}\right) \leqq h$ for any $F \in \Delta^{+}$and any $h \in[0,1]$.

LEMMA 2.4. If $\tau$ is a continuous triangle function, then for every $\varepsilon>0$ there is a $\delta_{0}>0$ such that for all $\delta \leqq \delta_{0}$ and for all $(F, G) \in \Delta^{+} \times \Delta^{+}$,

$$
\mathscr{L}\left(\tau(F, G), \tau\left(F^{\delta}, G^{\delta}\right)\right)<\varepsilon ;
$$

i.e.,

$$
\tau(F, G ; x+\varepsilon)+\varepsilon \geqq \tau\left(F^{\delta}, G^{\delta} ; x\right), \text { for } \quad x \in\left[0, \frac{1}{\varepsilon}\right),
$$

and

$$
\tau(F, G ; x-\varepsilon)-\varepsilon \leqq \tau\left(F^{\delta}, G^{\delta} ; x\right), \text { for } x \in\left[0, \frac{1}{\varepsilon}+\varepsilon\right) .
$$

Proof. Since $\left(\Delta^{+}, \mathscr{L}\right)$ is compact and since $\tau$ is continuous, $\tau$ is uniformly continuous.

LemMa 2.5. Let $(S, \mathscr{F})$ be a semi-PM space; let $\phi$ be a profile function, and let $p, q \in S$. Then $q \in N_{p}(\phi, \varepsilon)$ if and only if $F_{p q}^{\varepsilon} \geqq \phi$.

Proof. This follows immediately from Definition 2.1.

THEOREM 2.6. Let $(S, \mathscr{F})$ be a semi-PM space, let $p, q \in S$ and let $\phi$ be a profile function. Then 


$$
q \in C_{\phi}\{p\} \text { if and only if } F_{p q} \geqq \phi \text {. }
$$

Proof. By (2.1),

(2.3) $q \in C_{\phi}\{p\}$ if and only if $p \in N_{q}(\phi, \varepsilon)$ for every $\varepsilon>0$.

This, combined with Lemma 2.5, yields

$$
q \in C_{\phi}\{p\} \text { if and only if } F_{p q}^{\varepsilon} \geqq \phi \text { for every } \varepsilon>0 \text {. }
$$

But, since $F_{p q}$ is nondecreasing and left-continuous, it follows that $F_{p q}^{\varepsilon} \geqq \phi$ for every $\varepsilon>0$ if and only if $F_{p q} \geqq \phi$.

An immediate consequence of Theorem 2.6 is the following necessary and sufficient condition for the neighborhood system $\mathscr{N}_{\phi}$ to be $T_{1}$. This was obtained previously, first by E. Thorp [13; Theorem 3.4] and subsequently by R. Fritsche [6; Theorem 3.3].

Corollary 2.7. Let $(S, \mathscr{F})$ be a semi-PM space, and let $\phi$ be a profile function. The neighborhood system $\mathscr{N}_{\phi}$ is $T_{1}$ if and only if for each pair of points $p, q \in S, p \neq q$, there is an $x_{p q}>0$ such that $F_{p q}\left(x_{p q}\right)<\phi\left(x_{p q}\right)$.

The next theorem yields an interesting and useful connection between the neighborhood structures induced by two profile functions $\phi$ and $\psi$.

THEOREM 2.8. Let $(S, \mathscr{F}, \tau)$ be a PM space with a continuous triangle function $\tau$, and let $\phi$ and $\psi$ be profile functions. Then for every $p \in S$ and for every $\varepsilon>0$ there is $a \delta>0$ such that

$$
q \in N_{p}(\psi, \delta) \text { implies } N_{q}(\phi, \delta) \subset N_{p}(\tau(\phi, \psi), \varepsilon) \text {. }
$$

Proof. Let $\varepsilon>0$ be given. By Lemma 2.4 there is a $\delta>0$ such that for all $F, G \in \Delta^{+}$

$$
\tau(F, G ; x+\varepsilon)+\varepsilon \geqq \tau\left(F^{\delta}, G^{\delta} ; x\right), \text { for } x \in\left[0, \frac{1}{\varepsilon}\right) \text {. }
$$

If $q \in N_{p}(\psi, \delta)$ and if $r \in N_{q}(\phi, \delta)$, then by Lemma 2.5,

$$
F_{p q}^{\delta} \geqq \psi \text { and } \quad F_{q r}^{\delta} \geqq \phi
$$

From (2.6), (2.7), and the triangle inequality, it follows that for $x \in[0,1 / \varepsilon)$

$$
\begin{aligned}
F_{p r}(x+\varepsilon)+\varepsilon & \geqq \tau\left(F_{p q}, F_{q r} ; x+\varepsilon\right)+\varepsilon \\
& \geqq \tau\left(F_{p q}^{\delta}, F_{q r}^{\delta} ; x\right) \geqq \tau(\phi, \psi ; x) .
\end{aligned}
$$

Whence, $r \in N_{p}(\tau(\phi, \psi), \varepsilon)$ and $N_{q}(\phi, \delta) \subset N_{p}(\tau(\phi, \psi), \varepsilon)$. 
THEOREM 2.9. Under the hypotheses of Theorem 2.8, for every $A \subset S$,

$$
C_{\psi}\left(C_{\phi}(A)\right) \subset C_{\tau(\psi, \phi)}(A) .
$$

Proof. Let $p \in C_{\psi}\left(C_{\phi}(A)\right)$, and let $\varepsilon>0$ be given. By Theorem 2.8, there is a $\delta>0$ such that (2.5) holds. Since $p \in C_{\psi}\left(C_{\phi}(A)\right)$, there is a $q \in N_{p}(\psi, \delta) \cap C_{\rho}(A)$. Since $q \in N_{p}(\psi, \delta)$, our choice of $\delta$ yields:

$$
N_{q}(\phi, \delta) \subset N_{p}(\tau(\psi, \phi), \varepsilon) .
$$

But $q \in C_{\varphi}(A)$, so that $N_{q}(\phi, \delta) \cap A \neq \square$.

An inclusion similar to (2.8) occurs in the definition of a probabilistic topological space [4], where $\left(A^{\mu}\right)^{\lambda}$ is required to be contained in $A^{T(\lambda, \mu)}$. Example 2.13 shows that the inclusion (2.8) can be proper, and thus the result of this Theorem is best possible.

We now consider necessary conditions for $C_{\phi}$ to be idempotent.

Corollary 2.10. Let $(S, \mathscr{F}, \tau)$ be a PM space, and let $\phi$ be a profile function. If $\tau$ is continuous and $\tau(\phi, \phi)=\phi$, then $C_{\dot{\phi}}$ is a Kuratowski closure operator.

Proof. By (2) of Definition 1.4, Theorem 2.9, and the hypothesis that $\tau(\phi, \phi)=\phi$, for every $A \subset S$

$$
C_{\phi}(A) \subset C_{\phi}\left(C_{\phi}(A)\right) \subset C_{\tau(\phi, \phi)}(A)=C_{\phi}(A) .
$$

Thus $C_{\phi}$ is idempotent and the conclusion now follows.

Letting $\phi=\varepsilon_{0}$ yields the following result:

Corollary 2.11. Let $(S, \mathscr{F}, \tau)$ be a PM space. If $\tau$ is continuous, then $C_{\varepsilon_{0}}$ is a Kuratowski closure operator.

The conclusion of Corollary 2.10 can also be formulated in terms of neighborhoods:

CoRollary 2.12. Let $(S, \mathscr{F}, \tau)$ be a PM space, and let $\phi$ be a profile function. If $\tau$ is continuous and $\tau(\phi, \phi)=\phi$, then the neighborhood system $\mathscr{N}_{\phi}$ satisfies (nbd 4); i.e., for any $p \in S$ and $\varepsilon>0$, there is a $\delta>0$ such that $q \in N_{p}(\phi, \delta)$ implies $N_{q}(\phi, \delta) \subset N_{p}(\phi, \varepsilon)$.

Corollary 2.12 generalizes Theorem 7.2 of B. Schweizer and A. Sklar [9], (the case $\phi=\varepsilon_{0}$ ); and also both Theorem 3.13 of E. Thorp [13] and Theorem 3.2 of R. Fritsche [6], (the case $\phi=k \varepsilon_{0}, 0<k \leqq 1$ ). The next example shows that the result of Corollary 2.10 is 
best-possible for continuous triangle functions.

EXAMPLE 2.13. Let $S$ be the set of real numbers endowed with the Euclidean metric $d$; let $\mathscr{F}: S \times S \rightarrow \Delta^{+}$be given by $F_{p q}(x)=$ $\varepsilon_{d(p, q)}(x)$; and let $\tau=\tau_{T_{m}}$. It is easily verified that $(S, \mathscr{F}, \tau)$ is a PM space. Let $\phi(x)=1 / 2 \varepsilon_{1 / 2}(x)$ and note that

$$
\begin{aligned}
C_{\phi}\{p\} & =\{q \mid d(p, q) \leqq 1 / 2\} ; \\
C_{\phi} C_{\phi}\{p\} & =\{q \mid d(p, q) \leqq 1\} ; \quad C_{\tau(\phi, \phi)}\{p\}=S .
\end{aligned}
$$

(This last equality follows from $\tau(\phi, \phi)=\varepsilon_{\infty} \equiv 0$.)

Thus $C_{\phi}\{p\} \varsubsetneqq C_{\phi}\left(C_{\phi}\{p\}\right) \varsubsetneqq C_{\tau(\phi, \phi)}\{p\}$.

THeOREM 2.14. Let $(S, \mathscr{F}, \tau)$ be a PM space, and let $\phi$ be a profile function. If $\tau$ is continuous and if $\tau(\phi, \phi)=\phi$, then the closure structure induced by $C_{\phi}$ is pseudo-metrizable. If $C_{\phi}\{p\}=\{p\}$ for every $p \in S$, then it is metrizable.

Proof. For each natural number $n$ let $V_{n}=\{(p, q) \in S \times S \mid$ $F_{p q}(x+\lambda(n))+\lambda(n) \geqq \phi(x)$, for $\left.x \in[0,1 / \lambda(n))\right\}$, where $\lambda(n)$ is defined as follows: $\lambda(1)=1$. If $\lambda(n)$ has been defined, then by Theorem 2.8 there is a $\delta_{n}>0$ such that for every $p \in S$ and all $\delta<\delta_{n} q \in N_{p}(\phi, \delta)$ implies that $N_{q}(\phi, \delta) \subset N_{p}(\phi, \lambda(n))$. Define $\lambda(n+1)=\operatorname{Min}\left(\delta_{n}, 2^{-(n+1)}\right)$. It then follows that $V_{n}=V_{n}^{-1}$ and $V_{n+1} \circ V_{n+1} \subset V_{n}$.

This theorem generalizes Theorem 2 of B. Schweizer, A. Sklar, and E. Thorp [11] (where $\phi=\varepsilon_{0}$ ), Theorem 3.14 of E. Thorp [13], and Theorem 3.2 of R. Fritsche [6] (where $\phi=k \varepsilon_{0}, 0<k \leqq 1$ ).

THEOREM 2.15. Let $(S, \mathscr{F}, \tau)$ be a PM space, and let $\phi$ be a profile function. If $\tau$ is continuous, and if $\mathscr{N}_{\tau(\phi, \phi)}$ is $T_{1}$, then $\mathscr{N}_{\phi}$ is $T_{2}$.

Proof. Let $p, q \in S$, and suppose $p \neq q$. Since $\mathscr{N}_{\tau(\phi, \phi)}$ is $T_{1}$, there is an $\varepsilon>0$ such that $q \notin N_{p}(\tau(\phi, \phi), \varepsilon)$. By Theorem 2.8 there is a $\delta>0$ such that

$$
r \in N_{p}(\phi, \delta) \text { implies } N_{r}(\phi, \delta) \subset N_{p}(\tau(\phi, \phi), \varepsilon) .
$$

Suppose $r \in N_{p}(\phi, \delta) \cap N_{q}(\phi, \delta)$. Since $F_{q r}=F_{r q}, \quad r \in N_{q}(\phi, \delta)$ implies $q \in N_{r}(\phi, \delta)$. Thus by (2.9), $q \in N_{p}(\tau(\phi, \phi), \varepsilon)$. This is a contradiction. Thus $N_{p}(\phi, \delta) \cap N_{q}(\phi, \delta)=\square$ and $\mathscr{N}_{\phi}$ is $T_{2}$.

Theorem 2.15 merits comparison with Theorem 7.2 of [10], Theorem 3.7 of [13], and Theorem 2.2 of [6].

APPENDIX A. Different Topologies for $\Delta^{+}$. 
In addition to the modified Lévy metric $\mathscr{L}$ defined in (1.3), two other metrics for $\Delta^{+}$are of interest. The first is the usual Lévy metric which is given by

$$
\begin{gathered}
L(F, G)=\inf \{h \mid F(x+h)+h \geqq G(x) \geqq F(x-h)-h, \text { for } \\
\text { every } \times \in \boldsymbol{R}\} .
\end{gathered}
$$

The second is the usual sup norm which is given by

$$
\begin{gathered}
\Sigma(F, G)=\inf \{h \mid F(x)+h \geqq G(x) \geqq F(x)-h \text { for } \\
\text { every } x \in R\} .
\end{gathered}
$$

Each of these metrics could have been used to define a neighborhood structure for a semi-PM space; namely, by setting

$$
N_{p}^{L}(\dot{\phi}, \varepsilon)=\left\{q \in S \mid F_{p q}(x+h)+h \geqq \dot{\phi}(x) \text { for every } x\right\}
$$

and

$$
N_{p}^{\Sigma}(\phi, \varepsilon)=\left\{q \in S \mid F_{p q}(x)+h \geqq \dot{\phi}(x) \text { for every } x\right\} \text {. }
$$

It is clear that $N_{p}^{s}(\phi, \varepsilon) \subset N_{p}^{L}(\phi, \varepsilon) \subset N_{p}(\phi, \varepsilon)$ for every $\varepsilon>0$, and that each inclusion can be strict. Both of these neighborhood structures will satisfy (nbd 1), (nbd 2), and (nbd 3) and hence induce a closure space structure on the underlying space. The reason for choosing the metric $\mathscr{L}$ is that $\left(\Delta^{+}, \mathscr{L}\right)$ is compact, whereas neither $\left(\Delta^{+}, L\right)$ nor $\left(\Delta^{+}, \Sigma\right)$ is [7]. Thus, in these spaces, continuity of the triangle function is not enough to guarantee uniform continuity. Note that uniform continuity of the triangle function is used in the proof of Lemma 2.4 and that this lemma plays a crucial role in much of the subsequent development.

APPendix B. Comparison with the Work of E. Thorp and $R$. Fritsche.

The neighborhood structure for a semi-PM space given in Definition 2.1 is different from that given by E. Thorp in [13] and that given by R. Fritsche in [6]. Since R. Fritsche has shown that his neighborhood structure is essentially that of $\mathrm{E}$. Thorp, and since his definition more closely resembles Definition 2.1, we shall only consider the neighborhood structure given by him. R. Fritsche defines a $(\phi ; \varepsilon, \lambda)$-neighborhood of a point $p$ to be the set

$$
N_{p}(\phi ; \varepsilon, \lambda)=\left\{q \in S \mid F_{p q}(\varepsilon)>\phi(\varepsilon)-\lambda\right\} .
$$

The resultant neighborhood structure satisfies (nbd 1) and (nbd 2), but in general fails to satisfy (nbd 3). A necessary condition for 
(nbd 3), given in Theorem 2.2 of [6], is that $F_{p q}-\phi$ be nondecreasing for every pair $p, q \in S$. In this case we have:

THEOREM B. Let $(S, \mathscr{F})$ be a semi-PM space, and let $\dot{\phi}$ be a profile function. If $F_{p q}-\phi$ is nondecreasing for every pair of points $p, q \in S$, then for every $p \in S$, the neighborhood system $\mathscr{N}_{\phi}(p)$ is equivalent to the $(\phi ; \varepsilon, \lambda)$-neighborhood system at $P$.

REMARK. When $\dot{\phi}=\varepsilon_{0}$, the $(\phi ; \varepsilon, \lambda)$-neighborhood system is the $(\varepsilon, \lambda)$-neighborhood system studied by B. Schweizer and A. Sklar [9]. Since $F_{p q}-\varepsilon_{0}$ is always a nondecreasing function, it follows from Theorem $B$ that the $(\varepsilon, \lambda)$-neighborhood system is equivalent to the $\left(\varepsilon_{0}, \varepsilon\right)$-neighborhood system.

\section{Products of semi-PM spaces.}

Definition 3.1. Let $\left(S_{1}, \mathscr{F}_{1}\right)$ and $\left(S_{2}, \mathscr{F}_{2}\right)$ be semi-PM spaces, and let $\sigma$ be a triangle function. The $\sigma$-product of $\left(S_{1}, \mathscr{F}_{1}\right)$ and $\left(S_{2}, \mathscr{F}_{2}\right)$ is the pair $\left(S_{1} \times S_{2}, \mathscr{F}_{1} \times{ }_{\sigma} \mathscr{F}_{2}\right)$. Here $S_{1} \times S_{2}$ is the Cartesian product of $S_{1}$ and $S_{2}$, and $\mathscr{F}_{1} \times{ }_{\sigma} \mathscr{F}_{2}$ is the mapping from $\left(S_{1} \times S_{2}\right) \times\left(S_{1} \times S_{2}\right)$ into $\Delta^{+}$given by:

$$
\mathscr{F}_{1} \times \mathscr{F}_{2}(\vec{p}, \vec{q})=F_{\vec{p} \vec{q}}^{\sigma}=\sigma\left(F_{p_{1} q_{1}}, F_{p_{2} q_{2}}\right),
$$

where $\vec{p}=\left(p_{1}, p_{2}\right)$ and $\vec{q}=\left(q_{1}, q_{2}\right)$ belong to $S_{1} \times S_{2}$. (When there is no ambiguity, we shall denote $F_{\vec{p} \vec{q}}^{\sigma}$ by $F_{p q}$.)

If $\sigma=\Pi_{T}$ (viz. (1.5)), then the $\sigma$-product of two semi-PM spaces is the $T$-product as defined by R.J. Egbert in [3].

Definition 3.2. If $\left(S_{1}, \mathscr{F}_{1}\right)$ and $\left(S_{2}, \mathscr{F}_{2}\right)$ are semi-PM spaces, then $\left(S_{1}, \mathscr{F}_{1}\right)$ is isometric to $\left(S_{2}, \mathscr{F}_{2}\right)$ if there is a bijection $M: S_{1} \rightarrow S_{2}$ such that $F_{p q}=F_{M(p) M(q)}$ for every $p, q \in S_{1}$. The mapping $M$ is called an isometry.

The fact that the $\sigma$ in Definition 3.1 is a triangle function ensures that the $\sigma$-product of two semi-PM spaces has several natural properties. We enumerate these in:

THEOREM 3.3. Let $\left(S_{1}, \mathscr{F}_{1}\right)$ and $\left(S_{2}, \mathscr{F}_{2}\right)$ be semi-PM spaces. Then

(1) $\left(S_{1} \times S_{2}, \mathscr{F}_{1} \times{ }_{0} \mathscr{F}_{2}\right)$ is a semi-PM space;

(2) $\left(S_{1} \times S_{2}, \mathscr{F}_{1} \times \mathscr{F}_{2}\right)$ is isometric to $\left(S_{2} \times S_{1}, \mathscr{F}_{2} \times{ }_{\sigma} \mathscr{F}_{1}\right)$ under the natural map $M\left(p_{1}, p_{2}\right)=\left(p_{2}, p_{1}\right)$.

(3) For every $q_{2} \in S_{2}$, the natural $\operatorname{map} M_{q_{2}}: S_{1} \rightarrow S_{1} \times S_{2}$ given by $M_{q_{2}}(p)=\left(p, q_{2}\right)$ maps $\left(S_{1}, \mathscr{F}_{1}\right)$ isometrically onto the range of $M_{q_{2}}$. 
The associativity of $\sigma$ is not needed in the proof of Theorem 3.3. We require $\sigma$ to be associative so that we can unambiguously extend Definition 3.1 to $\sigma$-products of a finite number of semi-PM spaces.

We next direct our attention to finding necessary conditions for the $\sigma$-product of two PM spaces to be a PM space. We begin with:

Definition 3.4. Let $\sigma$ and $\tau$ be triangle functions. Then:

(i) $\sigma$ is stronger than $\tau$ (written $\sigma \geqq \tau$ ), if $\sigma(F, G) \geqq \tau(F, G)$ for all $(F, G) \in \Delta^{+} \times \Delta^{+}$.

(ii) $\sigma$ dominates $\tau$ (written $\sigma \gg \tau$ ), if $\sigma\left(\tau\left(F_{1}, G_{1}\right), \tau\left(F_{2}, G_{2}\right)\right) \geqq$ $\tau\left(\sigma\left(F_{1}, F_{2}\right), \sigma\left(G_{1}, G_{2}\right)\right)$, for all $F_{1}, F_{2}, G_{1}$, and $G_{2}$ in $\Delta^{+}$.

Letting $G_{1}=F_{2}=\varepsilon_{0}$ in (ii) shows that if $\sigma \gg \tau$, then $\sigma \geqq \tau$. The converse is false.

TheOREM 3.5. Let $\left(S_{1}, \mathscr{F}_{1}, \tau\right)$ and $\left(S_{2}, \mathscr{F}_{2}, \tau\right)$ be PM spaces under the same triangle function $\tau$, and let $\sigma$ be a triangle function which dominates $\tau$. Then the $\sigma$-product of $\left(S_{1}, \mathscr{F}_{1}\right)$ and $\left(S_{2}, \mathscr{F}_{2}\right)$ is a PM space under $\tau$.

Proof. In view of Theorem 3.3, we need only establish the triangle inequality. To this end, let $p=\left(p_{1}, p_{2}\right), q=\left(q_{1}, q_{2}\right)$, and $r=\left(r_{1}, r_{2}\right)$ be in $S_{1} \times S_{2}$. Since $\sigma \gg \tau$ and since both $\left(S_{1}, \mathscr{F}_{1}, \tau\right)$ and $\left(S_{2}, \mathscr{F}_{2}, \tau\right)$ are PM spaces we have,

$$
\begin{aligned}
F_{p r} & =\sigma\left(F_{p_{1} r_{1}}, F_{p_{2} r_{2}}\right) \geqq \sigma\left(\tau\left(F_{p_{1} q_{1}}, F_{q_{1} r_{1}}\right), \tau\left(F_{p_{2} q_{2}}, F_{q_{2} r_{2}}\right)\right) \\
& \geqq \tau\left(\sigma\left(F_{p_{1} q_{1}}, F_{p_{2} q_{2}}\right), \sigma\left(F_{q_{1} r_{1}}, F_{q_{2} r_{2}}\right)\right)=\tau\left(F_{p q}, F_{q r}\right) .
\end{aligned}
$$

(If $\left(X_{1}, d_{1}\right)$ and $\left(X_{2}, d_{2}\right)$ are metric spaces, then in order to define a well-behaved metric on the Cartesian product of $X_{1}$ and $X_{2}$ a two place function $f$ mapping $\boldsymbol{R}^{+} \times \boldsymbol{R}^{+} \rightarrow \boldsymbol{R}^{+}$is required which satisfies the following properties:

(1) $f(a, 0)=a$,

(2) $f(a, b)=f(b, a)$,

(3) $f(a, b) \geqq f(c, b)$, whenever $a \geqq c$,

(4) $f(a, f(b, c))=f(f(a, b), c)$,

(5) $f\left(a_{1}+b_{1}, a_{2}+b_{2}\right) \leqq f\left(a_{1}, a_{2}\right)+f\left(b_{1}, b_{2}\right)$.

Note that condition (5) states that $f$ dominates addition. In particular if $f(a, b)=\left(a^{p}+b^{p}\right)^{1 / p}, p \geqq 1$, then (5) is the familiar Minkowski Inequality.)

Corollary 3.6. Let $\left(S_{1}, \mathscr{F}_{1}, \tau\right)$ and $\left(S_{2}, \mathscr{F}_{2}, \tau\right)$ be PM spaces. If $\sigma=\pi_{\mathrm{Min}}$, if $\sigma=\tau$, or if $\tau=\tau_{T}$ and $\sigma=\pi_{T}$ (for some $t$-norm $T$ ), then the $\sigma$-product of $\left(S_{1}, \mathscr{F}_{1}\right)$ and $\left(S_{2}, \mathscr{F}_{2}\right)$ is a PM space under $\tau$. 
Proof. For any triangle function $\tau, \pi_{\text {Min }} \gg \tau$ and $\tau \gg \tau$; and for any $t$-norm $T, \pi_{T} \gg \tau_{T}$. (This last result is due to R.J. Egbert [3, Theorem 2].)

It can also be shown that the result of Theorem 3.5 is bestpossible in the sense that if $\sigma$ and $\tau$ are triangle functions and $\sigma$ does not dominate $\tau$, then there exist PM spaces $\left(S_{1}, \mathscr{F}_{1}, \tau\right)$ and $\left(S_{2}, \mathscr{F}_{2}, \tau\right)$ whose $\sigma$-product is not a PM space under $\tau$.

We close this section with several results-stated without proofconcerning the relationship between the neighborhood systems $\mathscr{N}_{\dot{\phi}}^{\mathscr{F}_{1} \times \mathscr{F}_{2}}$ and $\mathscr{N}_{\phi}^{\mathscr{F}_{1}} \times \mathscr{N}_{\dot{\phi}}^{\mathscr{F}_{2}}$, where $\mathscr{F}_{1} \times \mathscr{F}_{2}$ denotes $\mathscr{F}_{1} \times \mathscr{F}_{2}$.

ThEOREM 3.7. Let $\left(S_{1}, \mathscr{F}_{1}\right)$ and $\left(S_{2}, \mathscr{F}_{2}\right)$ be semi-PM spaces and let $\phi$ and $\psi$ be profile functions. Then:

(1) For any triangle function $\sigma \mathscr{N}_{\phi}^{\sigma_{1} \times \mathscr{F}_{2}}$ is finer than $\mathscr{N}_{\phi}^{F_{1}} \times \mathscr{N}_{\phi}^{F^{2}}$.

(2) If $\sigma$ is continuous then $\mathscr{N}_{\phi}^{\sigma_{1}} \times \mathscr{N}_{\phi}^{\sigma_{2}}$ is finer than $\mathscr{N}_{\sigma(\dot{\phi}, \dot{\phi})}^{\mathbb{F}, \mathscr{F}_{2}}$

(3) If in addition $\sigma(\phi, \phi)=\phi$, then $\mathscr{N}_{\phi}^{\mathscr{F}_{1}} \times \mathscr{N}_{\phi}^{\mathscr{F}_{2}}$ is equivalent to $\mathscr{N}_{9}^{\sigma_{1} \times \mathscr{T}_{2}}$.

4. The probabilistic diameter and the probabilistic Hausdorff metric.

DEFinition 4.1. Let $\left(F_{\lambda} ; \lambda \in \Lambda\right)$ be a family of functions in $\Delta^{+}$. The functions $\sup _{\lambda \in A} F_{\lambda}$ and $\inf _{\lambda \in \Lambda} F_{\lambda}$ are defined by:

(1) $\left(\sup _{\lambda \in A} F_{\lambda}\right)(x)=\sup _{\lambda \in A} F_{\lambda}(x)$, for each $x \in R$;

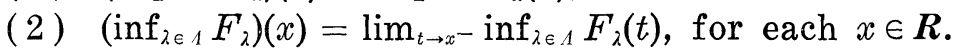

It is easily shown that $\sup _{\lambda \in A} F_{\lambda}$ is in $\Delta^{+}$; and $\inf _{\lambda \in A} F_{\lambda}$ is in $\Delta^{+}$by definition.

Definition 4.2. Let $(S, \mathscr{F})$ be a semi-PM space, and let $A$ be a nonempty subset of $S$. The probabilistic diameter $D_{A}$ of $A$ is given by $D_{A}=\inf _{p, q \in A} F_{p q}$.

Theorem 4.3. Let $(S, \mathscr{F})$ be a semi-PM space, and let $A$ and $B$ be nonempty subsets of $S$.

(1) If $A \subset B$, then $D_{A} \geqq D_{B}$.

(2) $D_{A}=\varepsilon_{0}$ if and only if $A=\{p\}$, for some $p \in S$.

(3) If there is a triangle function $\tau$ such that $(S, \mathscr{F}, \tau)$ is a PM space and if $A \cap B \neq \square$, then $D_{A \cup B} \geqq \tau\left(D_{A}, D_{B}\right)$.

Proof. The proof is similar to the proofs of Theorems 8, 9, and 10 of [3]. 
THEOREM 4.4. Let $(S, \mathscr{F})$ be a semi-PM space, let $\phi$ be a profile function, and let $A$ be a nonempty subset of $S$. Then (1) $D_{A} \geqq D_{C_{\phi}}(A)$. (2) If there is a continuous triangle function $\tau$ such that $(S, \mathscr{F}, \tau)$ is a $\mathrm{PM}$ space, then

$$
D_{C_{\phi}}(A) \geqq \tau\left(\tau\left(\phi, D_{A}\right), \phi\right)=\tau\left(\tau(\phi, \phi), D_{A}\right) .
$$

Proof. Since $A \subset C_{\phi}(A)$, (1) follows immediately from Theorem 4.3 .

Next, suppose $\tau$ is a continuous triangle function such that $(S, \mathscr{F}, \tau)$ is a PM space, and let $0<\varepsilon<1$. Since $\tau$ is continuous, Lemma 2.4 implies there is a $\delta>0$ such that for all $F, G, H \in \Delta^{+}$

$$
\tau(\tau(F, G), H ; x) \geqq \tau\left(\tau\left(F^{\delta}, G\right), H^{\delta} ; x-\varepsilon\right)-\varepsilon, \text { for } x \in\left[0, \frac{1}{\varepsilon}-\varepsilon\right) .
$$

Let $p, q \in C_{\phi}(A)$. Then $N_{p}(\phi, \delta) \cap A \neq \square$ and $N_{q}(\phi, \delta) \cap A \neq \square$; whence it follows from Lemma 2.5 that there exist $r$ and $s$ in $A$ such that

$$
F_{p r}^{\delta} \geqq \phi \quad \text { and } \quad F_{q s}^{\delta} \geqq \phi .
$$

From the triangle inequality and the relations $F_{r s} \geqq D_{A}$ and $F_{q s}=F_{s q}$, it follows that for $x \in[0,1 / \varepsilon-\varepsilon)$

$$
\begin{aligned}
F_{p q}(x) & \geqq \tau\left(\tau\left(F_{p r}, F_{r s}\right), F_{s q} ; x\right) \\
& \geqq \tau\left(\tau\left(F_{p r}^{\delta}, F_{r s}\right), F_{s q}^{\delta} ; x-\varepsilon\right)-\varepsilon, \\
& \geqq \tau\left(\tau\left(\phi, D_{A}\right), \phi ; x-\varepsilon\right)-\varepsilon .
\end{aligned}
$$

Letting $\varepsilon \rightarrow 0$ and using the left-continuity of $\tau\left(\tau\left(\phi, D_{A}\right), \phi\right)$ yields

$$
F_{p q} \geqq \tau\left(\tau\left(\phi, D_{A}\right), \phi\right) \text { for any } p, q \in C_{\phi}(A) \text {. }
$$

Consequently,

$$
D_{C_{\phi}}(A) \geqq \tau\left(\tau\left(\phi, D_{A}\right), \phi\right)=\tau\left(\tau(\phi, \phi), D_{A}\right) .
$$

Letting $\phi=\varepsilon_{0}$, we obtain the following result, which is due to R.J. Egbert [3].

Corollary 4.5. Let $(S, \mathscr{F}, \tau)$ be a PM space. If $\tau$ is continuous, then $D_{A}=D_{C_{\varepsilon_{0}}}(A)$.

Definition 4.6. Let $(S, \mathscr{F})$ be a semi-PM space, let $A$ and $B$ be nonempty subsets of $S$, and let $\sigma$ be a triangle function. The probabilistic Hausdorff distance $(\bmod \sigma)$ between $A$ and $B$, denoted by $F_{A B}^{o}$, is the distribution function given by

$$
F_{A B}^{\sigma}=\sigma(A B, B A) \text {, where }
$$




$$
\left.A B(x)=\inf _{p \in A} \sup _{q \in B} F_{p q}\right)(x) .
$$

When there is no ambiguity, we shall suppress the reference to $\sigma$ and denote $F_{A B}^{o}$ by $F_{A B}$.

If $\sigma=\pi_{T}$, then $F_{A B}^{o}$ is the distance defined by R.J. Egbert [3]. The following are immediate:

THEOREM 4.7. Let $(S, \mathscr{F})$ be a semi-PM space, and let $A$ and $B$ be nonempty subsets of $S$. Then $F_{A A}=\varepsilon_{0}$ and $F_{A B}=F_{B A}$.

LeMMA 4.8. Let $(S, \mathscr{F})$ be a semi-PM space, let $\phi$ be a profile function, and let $A$ and $B$ be nonempty subsets of $S$. If $A B \geqq$ $\left(\sup _{t \in R} \phi(t)\right) \varepsilon_{0}=(\sup \phi) \varepsilon_{0}$, then $A \subset C_{\phi}(B)$.

TheOREM 4.9. Let $(S, \mathscr{F})$ be a semi-PM space, let $\phi$ be a profile function, and let $A$ and $B$ be nonempty subsets of $S$. If $F_{A B} \geqq$ $(\sup \phi) \varepsilon_{0}$, then

$$
A \subset C_{\phi}(B) \text { and } B \subset C_{\phi}(A) \text {. }
$$

The next result is a restatement of Theorem 16 of [3].

CoRollary 4.10. Let $(S, \mathscr{F}, \tau)$ be a PM space, and let $A$ and $B$ be nonempty subsets of $S$. If $\tau$ is a continuous triangle function, then

$$
F_{A B}=\varepsilon_{0} \quad \text { if and only if } C_{\varepsilon_{0}}(A)=C_{\varepsilon_{0}}(B) \text {. }
$$

Definition 4.11. Let $(S, \mathscr{F})$ be a semi-PM space, let $A$ and $B$ be nonempty subsets of $S$, and let $\phi$ be a profile function. Then $A$ is $\phi$-equivalent to $B$, if

$$
F_{C_{\phi}(A) C_{\phi}(B)}=\varepsilon_{0} .
$$

If follows immediately from Theorem 4.7 that $\phi$-equivalence is a reflexive and symmetric relation. The next theorem will yield sufficient conditions for this relation to be an equivalence relation. We begin with:

Definition 4.12. The triangle function $\tau$ is sup-continuous if, for every family of functions $\left(F_{\lambda} ; \lambda \in \Lambda\right)$ in $\Delta^{+}$and for every $H \in \Delta^{+}$,

$$
\tau\left(\sup _{\lambda \in A} F_{\lambda}, H\right)=\sup _{\lambda \in A} \tau\left(F_{\lambda}, H\right) .
$$

It follows immediately from the left-continuity of $T$ that $\pi_{T}$ and $\tau_{T}$ are sup-continuous. On the other hand, convolution is not sup-continuous. 
LEMMA 4.13. Let $(S, \mathscr{F}, \tau)$ be a PM space, and let $A, B$, and $C$ be nonempty subsets of $S$. If $\tau$ is sup-continuous, then $A B \geqq$ $\tau(A C, C B)$.

Proof. For any $p \in A, q \in B$, and $r \in C$

$$
F_{p q} \geqq \tau\left(F_{p r}, F_{r q}\right) \text {. }
$$

The sup-continuity of $\tau$ and (4.3) yield

$$
\sup _{q \in B} F_{p q} \geqq \tau\left(F_{p r}, \sup _{q \in B} F_{r q}\right), \text { for } p \in A, r \in C .
$$

Since $\sup _{q \in B} F_{r q} \geqq \inf _{r \in C} \sup _{q \in B} F_{r q}=C B$, (4.4) implies

$$
\sup _{q \in B} F_{p q} \geqq \tau\left(F_{p r}, C B\right), \text { for } p \in A, r \in C \text {. }
$$

Again, since $\tau$ is sup-continuous, (4.5) implies

$$
\sup _{q \in B} F_{p q} \geqq \tau\left(\sup _{r \in C} F_{p r}, C B\right), \text { for } \quad p \in A \text {. }
$$

And hence, (4.6) implies

$$
\begin{aligned}
A B & =\inf _{p \in A} \sup _{q \in B} F_{p q} \geqq \inf _{p \in A} \tau\left(\sup _{r \in C} F_{p r}, C B\right) \geqq \tau\left(\inf _{p \in A} \sup _{r \in C} F_{p r}, C B\right) \\
& =\tau(A C, C B) .
\end{aligned}
$$

TheOREM 4.14. Let $(S, \mathscr{F}, \tau)$ be a PM space, and let $A, B$, and $C$ be nonempty subsets of $S$. If $\sigma \gg \tau$ and if $\tau$ is sup-continuous, then

$$
F_{A B}^{\sigma} \geqq \tau\left(F_{A C}^{\sigma}, F_{C B}^{\sigma}\right)
$$

Proof. Using Lemma 4.13, the commutativity of $\tau$ and the fact that $\sigma \gg \tau$, we have:

$$
\begin{aligned}
F_{A B}^{o} & =\sigma(A B, B A) \geqq \sigma(\tau(A C, C B), \tau(B C, C A)) \\
& =\sigma(\tau(A C, C B), \tau(C A, B C)) \geqq \tau(\sigma(A C, C A), \sigma(C B, B C)) \\
& =\tau\left(F_{A C}^{o}, F_{C B}^{o}\right) .
\end{aligned}
$$

CoRollary 4.15. Let $(S, \mathscr{F}, \tau)$ be a PM space, and let $\phi$ be a profile function. If $\sigma \gg \tau$ and if $\tau$ is sup-continuous, then $\phi$-equivalence is an equivalence relation.

Theorem 4.16. Let $(S, \mathscr{F}, \tau)$ be a PM space, let $\phi$ be a profile function, and let $S_{\phi}=\left\{C_{\phi}(B) \mid B \subset S, B \neq \square\right\}$. Suppose that $\sigma \gg \tau$ and that $\tau$ is both sup-continuous and continuous. Then $\left(S_{\phi}, \mathscr{F}^{\sigma}, \tau\right)$ is a PM space. 
Proof. For $A, B$ and $C$ in $S_{\phi}$, by Theorems 4.1 and 4.14, we have: (a) $F_{A B}^{o}=\varepsilon_{0}$, if $A=B$; (b) $F_{B A}^{\sigma}=F_{A B}^{\sigma}$; (c) $F_{B A}^{\sigma} \geqq \tau\left(F_{A C}^{o}, F_{C B}^{\sigma}\right)$. Hence, all that remains to be shown is that if $F_{A B}=\varepsilon_{0}$, then $A=B$. By Corollary 4.10, $F_{A B}=\varepsilon_{0}$ implies

$$
C_{\varepsilon_{0}}(A)=C_{\varepsilon_{0}}(B) \text {. }
$$

Since $A, B \in S_{\phi}$, there exist $A^{\prime}, B^{\prime} \subset S$ such that $A=C_{\phi}\left(A^{\prime}\right)$ and $B=C_{\phi}\left(B^{\prime}\right)$. Consequently $\mathrm{C}_{\varepsilon_{0}} C_{\phi}\left(A^{\prime}\right)=C_{\varepsilon_{0}} C_{\phi}\left(B^{\prime}\right)$. By Theorem 2.9 and Definition 1.4

$$
\begin{aligned}
& C_{\phi}\left(A^{\prime}\right) \subset C_{\varepsilon_{0}} C_{\phi}\left(A^{\prime}\right) \subset C_{\tau\left(\varepsilon_{0}, \phi\right)}\left(A^{\prime}\right)=C_{\phi}\left(A^{\prime}\right) ; \\
& C_{\phi}\left(B^{\prime}\right) \subset C_{\varepsilon_{0}} C_{\phi}\left(B^{\prime}\right) \subset C_{\tau\left(\varepsilon_{0}, \phi\right)}\left(B^{\prime}\right)=C_{\phi}\left(B^{\prime}\right) .
\end{aligned}
$$

Whence, by (4.8),

$$
A=C_{\phi}\left(A^{\prime}\right)=C_{\varepsilon_{0}} C_{\phi}\left(A^{\prime}\right) C_{\varepsilon_{0}} C_{\phi}\left(B^{\prime}\right)=C_{\phi}\left(B^{\prime}\right)=B .
$$

In the special case when $\phi=\varepsilon_{0}, \sigma=\Pi_{T}$ and $\tau=\tau_{T}$, this is Theorem 18 of [3].

We conclude with the observation that under the hypothesis of Corollary 4.15 the relation

$$
p \sim_{\phi} q \Longleftrightarrow C_{\phi}\{p\}=C_{\phi}\{q\}
$$

defines an equivalence relation on the points of $S$. In [8] B. Schweizer defines a tolerance relation called "indistinguishable $\bmod \phi$ " by

$$
p \sim q(\bmod \phi) \Longleftrightarrow F_{p q} \geqq \phi .
$$

It is clear that $p \sim_{\phi} q$ implies $p \sim q(\bmod \phi)$. However, the converse is false. Example 2.13 shows that if $p=0$ and $q=1 / 4$, then $p \sim q(\bmod \phi)$, but $C_{\phi}\{p\} \neq C_{\phi}\{q\}$ whence $p \sim_{\phi} q$ is false.

\section{REFERENCES}

1. A. Appert and Ky-Fan, Espaces topologiques intermediaires, Actualites Scientifiques et industrielles, No. 1121, Hermann \& Cie, Paris, 1951.

2. E. Čech, Topological spaces, Interscience Publishers, New York, 1966.

3. R. J. Egbert, Products and quotients of probabilistic metric spaces, Pacific J. Math. 24 (1968), 437-455.

4. M. J. Frank, Probabilistic topological spaces, J. Math. Analysis and Appl. 34 (1971), 67-81.

5. M. Frechet, Les espances abstraits, Gauthier-Villars, Paris 1928.

6. R. Fritsche, Topologies for probabilistic metric spaces, Fundamenta Mathematicae, 72 (1971), 7-16.

7. B. Schweizer, Multiplication on the space of probability distribution functions, Aeq. Math., 12 (1975), 156-183.

8. B. Schweizer, Sur la possibilite de distinguer les points dans un espaces metrique aleatoires, C. R. Acad. Sci., Paris, 280, Series A, (1975), 459-461. 
9. B. Schweizer and A. Sklar, Statistical metric spaces, Pacific J. Math., 10 (1960), 313-334.

10. B. Schweizer and A. Sklar, Statistical metric spaces arising from sets of random variables in Euclidean $n$-space, Theoriya Veroyatnostei i ee Primeneniya, 7 (1962), 456-465.

11. B. Schweizer, A Sklar, and E. Thorp, The metrization of statistical metric spaces, Pacific J. Math. 10 (1960), 673-675.

12. D. A. Sibley, A metric for weak convergence of distribution functions, Rocky Mountain J. Math. 1 (1971), 427-430.

13. E. Thorp, Generalized topologies for statistical metric spaces, Fundamenta Mathematicae, 51 (1962), 9-21.

Received November 3, 1975 and in revised form February 9, 1976. The results are from the author's $\mathrm{Ph}$. D. dissertation written at the University of Massachusetts and directed by Professor B. Schweizer. The author wishes to thank Professor Schweizer for his helpful comments and criticisms.

University of Minnesota, MorRis 



\section{PACIFIC JOURNAL OF MATHEMATICS}

\section{EDITORS}

RICHARD ARENS (Managing Editor) University of California

Los Angeles, California 90024

R. A. Beaumont

University of Washington

Seattle, Washington 98105
J. DUGUNDJI

Department of Mathematics University of Southern Californı

Los Angeles, California 90007

D. Gilbarg and J. Milgram

Stanford University

Stanford, California 94305

\section{ASSOCIATE EDITORS}
E. F. BECKENBACH
B. H. NeumanN
F. WOLF
K. YosHIDA

\section{SUPPORTING INSTITUTIONS}

UNIVERSITY OF BRITISH COLUMBIA

CALIFORNIA INSTITUTE OF TECHNOLOGY

UNIVERSITY OF CALIFORNIA

MONTANA STATE UNIVERSITY

UNIVERSITY OF NEVADA

NEW MEXICO STATE UNIVERSITY

OREGON STATE UNIVERSITY

UNIVERSITY OF OREGON

OSAKA UNIVERSITY
UNIVERSITY OF SOUTHERN CALIFORNIA

STANFORD UNIVERSITY

UNIVERSITY OF TOKYO

UNIVERSITY OF UTAH

WASHINGTON STATE UNIVERSITY

UNIVERSITY OF WASHINGTON

* * * *

AMERICAN MATHEMATICAL SOCIETY

NAVAL WEAPONS CENTER 


\section{Pacific Journal of Mathematics \\ Vol. 65, No. $1 \quad$ September, 1976}

David Lee Armacost, Compactly cogenerated LCA groups ............. 1

Sun Man Chang, On continuous image averaging of probability measures ...... 13

J. Chidambaraswamy, Generalized Dedekind $\psi$-functions with respect to a

polynomial. II................................... 19

Freddy Delbaen, The Dunford-Pettis property for certain uniform algebras ..... 29

Robert Benjamin Feinberg, Faithful distributive modules over incidence

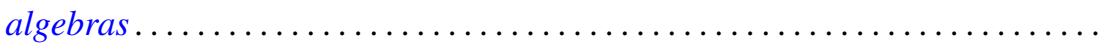

Paul Froeschl, Chained rings . . . . . . . . . . . . . . . . . . . .

John Brady Garnett and Anthony G. O'Farrell, Sobolev approximation by a sum

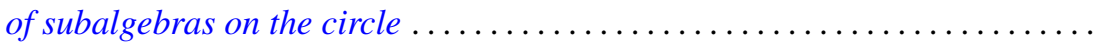

Hugh M. Hilden, José M. Montesinos and Thomas Lusk Thickstun, Closed

oriented 3-manifolds as 3-fold branched coverings of $S^{3}$ of special type.....

Atsushi Inoue, On a class of unbounded operator algebras ................

Peter Kleinschmidt, On facets with non-arbitrary shapes.

Narendrakumar Ramanlal Ladhawala, Absolute summability of Walsh-Fourier

series

Howard Wilson Lambert, Links which are unknottable by maps . . . . . . . . . . .

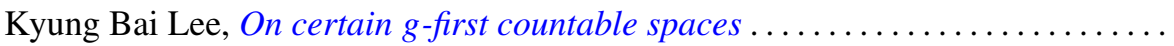

Richard Ira Loebl, A Hahn decomposition for linear maps .................

Moshe Marcus and Victor Julius Mizel, A characterization of functionals on $W_{1}^{p}$ possessing autonomous kernels. I . .

James Miller, Subordinating factor sequences and convex functions of several

variables.

Keith Pierce, Amalgamated sums of abelian l-groups ...

Jonathan Rosenberg, The $C^{*}$-algebras of some real and $p$-adic solvable

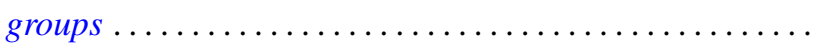

Hugo Rossi and Michele Vergne, Group representations on Hilbert spaces defined

in terms of $\partial_{b}$-cohomology on the Silov boundary of a Siegel domain . .

Mary Elizabeth Schaps, Nonsingular deformations of a determinantal

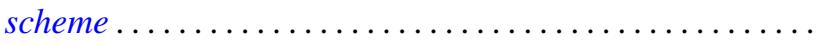

S. R. Singh, Some convergence properties of the Bubnov-Galerkin method...

Peggy Strait, Level crossing probabilities for a multi-parameter Brownian

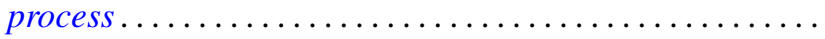

Robert M. Tardiff, Topologies for probabilistic metric spaces .

Benjamin Baxter Wells, Jr., Rearrangements of functions on the ring of integers of

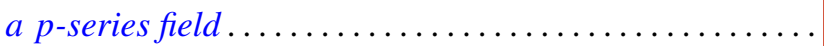

Robert Francis Wheeler, Well-behaved and totally bounded approximate identities for $C_{0}(X)$.

Delores Arletta Williams, Gauss sums and integral quadratic forms over local

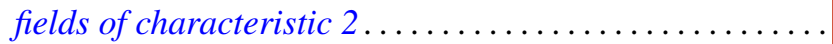

John Yuan, On the construction of one-parameter semigroups in topological 